\title{
Role of intrauterine instillation of levobupivacaine as a local anaesthetic for outpatient gynaecological procedures: a randomized controlled study
}

\author{
Taru Gupta ${ }^{1}$, Ankita Mansinghka ${ }^{1 *}$, Anil Thakur ${ }^{2}$ \\ ${ }^{1}$ Department of Obstetrics and Gynecology, ESI PGIMSR Basaidarapur, New Delhi, India \\ ${ }^{2}$ Department of Anaesthesia, ESI PGIMSR Basaidarapur, New Delhi, India
}

Received: 06 January 2020

Revised: 13 January 2020

Accepted: 18 January 2020

\section{*Correspondence:}

Dr. Ankita Mansinghka,

E-mail: ankita.mansinghka@gmail.com

Copyright: $\odot$ the author(s), publisher and licensee Medip Academy. This is an open-access article distributed under the terms of the Creative Commons Attribution Non-Commercial License, which permits unrestricted non-commercial use, distribution, and reproduction in any medium, provided the original work is properly cited.

\begin{abstract}
Background: Procedures such as intrauterine device (IUD) insertion and endometrial biopsy, are routinely performed in the outpatient setting for various indications. Satisfactory pain control for women undergoing outpatient gynecological procedures is critical for both patient comfort and procedure success. The perception of pain during gynecological procedures originates from manipulation of the cervix and/or uterus.

Methods: This study evaluated the role of intrauterine instillation of $0.5 \%$ levobupivacaine as local anesthetic in terms of pain score during and post outpatient gynecological procedures (endometrial aspiration biopsy and IUD insertion), reduction in need for post procedural analgesia and allowance of early return to normal activity. The trial medication was intrauterine anesthesia, either $5 \mathrm{~mL} 0.5 \%$ levobupivacaine or $5 \mathrm{~mL} 0.9 \%$ saline (control group) on 100 patients. Primary outcome was self-reported pain scores on numerical pain scale at various points of procedure, point (0) was a grade for as no pain and the worst pain was graded as ten point (10) in this scale. During the procedure, degree of pain was specified by study subjects at 4 steps. These steps were 1 ) immediately after tenaculum application, 2) during the solution instillation and 3) IUD insertion or EB and 4) 15 minutes after the procedure and 24 hours later.

Results: Pain scores of the intrauterine levobupivacaine groups were found to be significantly lower than the control group also there was reduced need for additional analgesia and also, they had early return to normal activity.

Conclusions: Study sought to reduce pain during OPD procedures which might lead to better patient experience, reduce anxiety and produce an overall better outcome.
\end{abstract}

Keywords: Analgesia, Intrauterine, Levobupivacaine, Pain

\section{INTRODUCTION}

A major obstacle to the successful completion of outpatient gynecologic procedures is pain management. Pain during and after the procedure is one of the causes of concern during uterine manipulation for these procedures and hence needs to be addressed. Common outpatient gynecological procedures like diagnostic endometrial biopsy (EB), intrauterine device insertion (IUD), are associated with pain and discomfort. Most women experience mild to moderate pain. Pain may occur during dilation of the cervix for insertion of the catheter and during endometrial biopsy, which further aggravates due to uterine contractions. ${ }^{1}$ Sometimes the 
pain is severe and can be associated with vasovagal reactions like light-headedness, nausea, syncope and very rarely convulsions.

Uterus and cervix are richly innervated with autonomic nerves arising from the S2-S4 roots and travel to the uterus in the lower portion of the broad ligament as the Franken Hauser plexus, the paracervical block aims to interrupt these nerves. The uterus has nerve supply originating from other points as well which can be blocked with intrauterine instillation of an anesthetic drug. Levobupivacaine has a less depressant effect on myocardium and central nervous system with a superior pharmacokinetic profile as compared to bupivacaine. ${ }^{2}$

This study evaluated the role of intrauterine instillation of $0.5 \%$ levobupivacaine as local anesthetic in terms of pain score during and post outpatient gynecological procedures, reduction in need for post procedural analgesia and allowance of early return to normal activity.

\section{METHODS}

The study design was a prospective, interventional double blinded comparative randomized control study conducted during the period of 18 months in the department of obstetrics and gynecology, E.S.I. PGIMSR Basaidarapur, New Delhi. Ethical clearance was taken from institution Ethical Committee. Hundred women with the complaints of abnormal uterine bleeding (AUB), infertility, postmenopausal bleeding (PMB) and in need of contraception, meeting the inclusion criteria were evaluated and counselled for procedure like IUD or EB were enrolled in the study after taking written informed consent. The study population was divided into two groups: Intervention group - $5 \mathrm{ml}$ of $0.50 \%(50 \mathrm{mg})$ levobupivacaine and Control group - $5 \mathrm{ml}$ of normal saline. Statistical testing was conducted with the statistical package for the social science system version SPSS 17.0. Continuous variables were presented as mean \pm SD or median if the data is unevenly distributed. Categorical variables were expressed as frequencies and percentages. For all statistical tests, a $\mathrm{p}$ value less than 0.05 was taken to indicate a significant difference.

Women age between 25-45 years requiring diagnostic EB or IUD insertion for contraception and therapeutic purpose were included in the study. Following were the exclusion criteria- absolute refusal, known or tested allergic to levobupivacaine, deranged kidney function test, pelvic inflammatory disease or acute cervicitis/vaginitis or such history within last 3 months, cervical malignancy, previous cervical surgery like loop electrosurgical excision or cryotherapy or cervical stenosis.

Information related the process of the IUD insertion and EB was given to all participants and awaiting them to score their ongoing agony level by using numerical rating scale (NRS) during the steps of the procedure. Random allocation of women into intervention and control groups by means sequentially numbered opaque slips envelopes was done. Assistant was asked to pick up an envelope and provide the drug filled canula to the investigator. Test dose or sensitivity testing for levobupivacaine was done before the start of procedure in all the patients with 0.2 $\mathrm{ml}$ of $0.5 \%$ levobupivacaine on forearm. Prior to the procedure, patient's demographic data age, gravidity, parity, Body mass index (BMI), and history of cesarean section was noted down. $5 \mathrm{~mL}$ of $0.9 \%$ saline for the control group, or $5 \mathrm{~mL}$ of $0.5 \%$ levobupivacaine for the study group was taken. Identical, colorless, unlabeled 10 $\mathrm{mL}$ disposable syringes were used to place the solutions into the uterine cavity. Primary investigator and study subjects were blinded to the type of solution. Under all asepsis routine pelvic examination was done followed by speculum insertion into the vagina to visualize cervix. The anterior lip of the cervix was grasped by tenaculum and was pulled slightly. Unlabeled test solution $(5 \mathrm{~mL})$ was instilled through the endocervix into the uterine cavity with the help of Karman's canula No 4. The Karman`s canula was stayed active for $15 \mathrm{~min}$ to permit the drug to produce results. After 15 minutes of waiting, IUD or EB was applied/performed in the standard method. Subjects were asked to score their ongoing agony level by using NRS during the steps of the procedure. Zero point (0) was a grade for as no pain and the worst pain was graded as ten point (10) in this scale. During the procedure, degree of pain was specified by study subjects at 4 steps. These steps were;

- Immediately after tenaculum application

- During the solution instillation and

- IUD insertion or EB and

- 15 minutes after the procedure.

Pulse rate, respiratory rate, BP was measured at these four steps. The patients were observed for $60 \mathrm{~min}$ in a recovery room and assessed for any side effects and complications. Patient were advised a course of antibiotics and analgesics (only to be taken if there is pain). Patient were contacted telephonically after 24 hours to assess the pain score, need of analgesic and time to return to normal activity.

\section{RESULTS}

Table 1: Patient profile in the study groups.

\begin{tabular}{|llll|}
\hline & $\begin{array}{l}\text { Participants } \\
\text { Group A } \\
\text { (cases) }\end{array}$ & $\begin{array}{l}\text { Group B } \\
\text { (control) }\end{array}$ & p value \\
& Mean \pm SD & Mean \pm SD & \\
\hline Age (years) & $40.16 \pm 7.16$ & $40.04 \pm 5.19$ & 0.923 \\
\hline Height $(\mathrm{cm})$ & $153.54 \pm 8.13$ & $153.20 \pm 6.15$ & 0.814 \\
\hline Weight $(\mathrm{kg})$ & $60.12 \pm 10.94$ & $58.88 \pm 10.72$ & 0.568 \\
\hline BMI $\left(\mathrm{kg} / \mathrm{m}^{2}\right)$ & $25.46 \pm 4.08$ & $25.07 \pm 4.39$ & 0.644 \\
\hline
\end{tabular}


In this study both the groups were demographically comparable in terms of age, weight, height, BMI, and education status (Table 1). We observed that mean pain score at step 1 , that is, immediately after tenaculum placement was 6.0 in Group A and 5.58 in Group B. (p value $=0.168)$ The mean pain score at step 2 , that is, during drug instillation was 6.08 in Group A and 6.26 in Group B $(\mathrm{p}$ value $=0.527)$. The mean pain score at step 3 , that is, during the procedure (IUD insertion or EB) was 4.10 in Group A and 6.78 in Group B. The difference in mean pain score at this step was statistically significant in two groups ( $\mathrm{p}$ value $<0.001$ ). The mean pain score at step 4, that is, 15 minutes after the procedure was 3.08 in Group A and 5.42 in Group B. (p value <0.001). This implies that there is significant reduction in pain score at step 3 and 4 in group which received intrauterine levobupivacaine (Table 2). It was also observed that mean pain score after 24 hours of procedure in Group A was 0.78 while that in Group B was 2.14. ( $p$ value $<0.001)$. Thus, pain score was significantly lower in levobupivacaine group even after 24 hours of the procedure (Figure 1)

Table 2: Comparison of pain score in between the two groups at various steps.

\begin{tabular}{|llll|}
\hline \multirow{2}{*}{ Pain score } & Participants & & Group B (control) \\
& Group A (cases) & p value \\
\hline Step 1-pain score (during tenaculum placement) & Mean \pm SD & Mean \pm SD & \\
\hline Step 2-pain score (drug instillation) & $6.00 \pm 1.59$ & $5.58 \pm 1.43$ & 0.168 \\
\hline Step 3-pain score (during procedure) & $6.08 \pm 1.48$ & $6.26 \pm 1.35$ & 0.527 \\
\hline Step 4-pain score (15 minutes after the procedure) & $4.10 \pm 1.31$ & $6.78 \pm 1.49$ & $<0.001$ \\
\hline Pain score 24 hour later & $3.08 \pm 1.32$ & $5.42 \pm 1.31$ & $<0.001$ \\
\hline
\end{tabular}

Step 1 - During tenaculum placement, Step 2 - During drug instillation, Step 3 - During procedure, Step 4 - 15 Minutes after the procedure

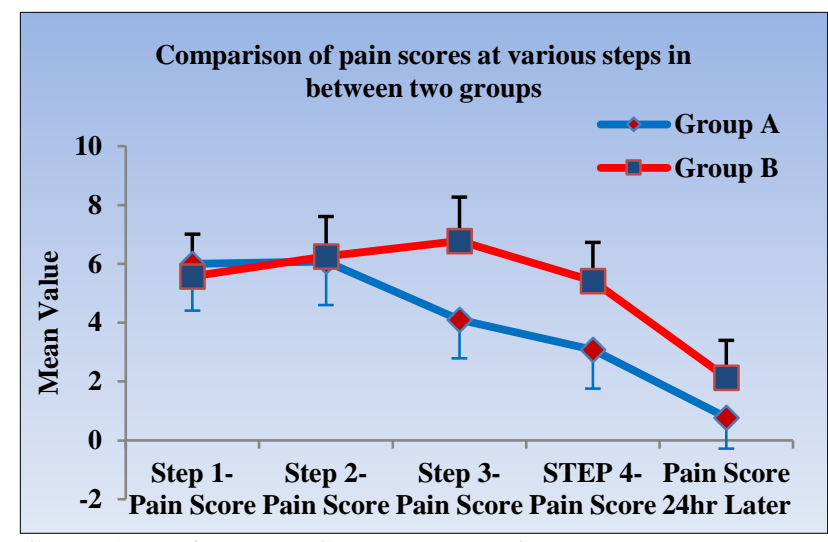

Group A: study group, Group B: control group.

Figure 1: The pain score in between the two groups.

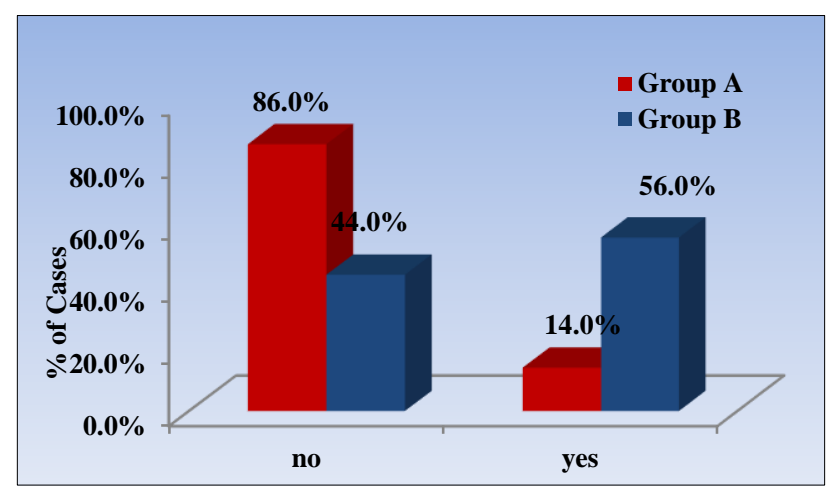

Group A: study group, Group B: control group.

Figure 2: Comparison of need for analgesia in between the two groups.

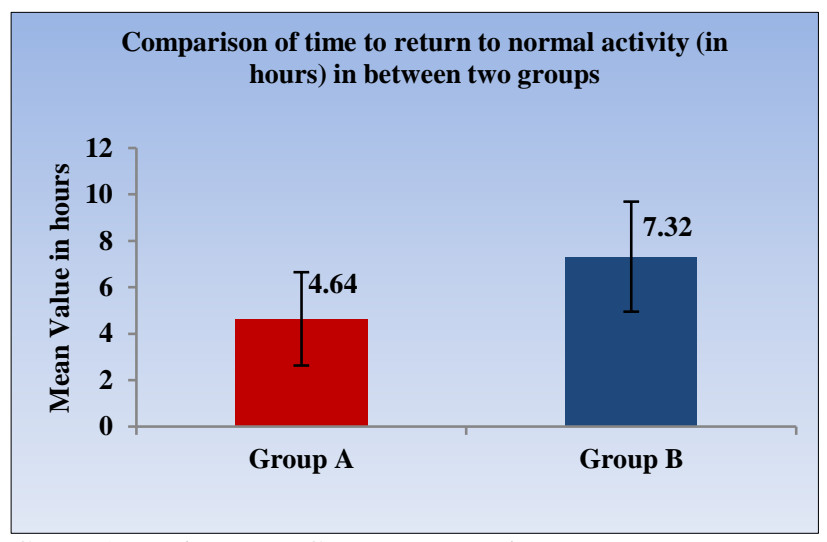

Group A: study group, Group B: control group.

Figure 3: Comparison of time required to return to normal activity in between the two groups.

Requirement of additional analgesia was also noted through a telephonic communication made with the subject after 24 hours of the procedure. In Group A, 43 $(86 \%)$ patients did not required any additional analgesia after discharge whereas in Group B, $28(56 \%)$ patients took analgesics after discharge ( $\mathrm{p}$ value $<0.001)$ (Figure 2 ). It was observed that patients in Group A had an early return to normal activity with mean time (in hours) of 4.64 hours while patients in Group B had delayed return to normal activity with time (in hours) of 7.32 hours. Thus, the patients who received intrauterine levobupivacaine as local anesthetic agent had an early return to normal activity ( $\mathrm{p}$ value $=0.001$ ) (Figure 3$)$. In this study authors also took into account the vitals of the 
patients such as pulse rate, blood pressure, respiratory rate at same four steps where pain was assessed. It was observed that there was no significant in between the two groups at all the four steps in values of blood pressure and respiratory rate, but there was significant difference in pulse rate after levobupivacaine instillation in Group A at the time of procedure (EB/IUD insertion) and step 4 which is, 15 minutes after the procedure as compared to Group B which received normal saline $(\mathrm{p}<0.001$ and $\mathrm{p}$ $<0.001$ respectively).

\section{DISCUSSION}

Various local anesthetics such as lidocaine, prilocaine in various forms such as spray, cream, gels, intrauterine instillation, paracervical blocks for pain control in various OPD procedures have been studied previously. In this study $0.5 \%$ of $5 \mathrm{ml}$ levobupivacaine was used as a local anesthetic agent for the study group and $5 \mathrm{ml}$ of $0.9 \%$ of normal saline was used in the control group. Both these solutions were colorless, thus avoiding any kind of biasing.

Akgun $\mathrm{N}$ et al, performed a similar study on 95 patients during IUD insertion comparing intrauterine instillation of levobupivacaine with control group and pain was assessed at similar four steps, with the help of visual analogue scale (VAS). ${ }^{3}$ The mean pain score using VAS during tenaculum placement and solution instillation showed no significant difference in between the two groups ( $\mathrm{p}$ values 0.105 and 0.241 respectively), while the mean pain score using VAS during IUD insertion and 15 minutes after the procedure was lower in study group as compared to control group ( $p$ value $<0.001$ and $<0.001$ respectively). Similarly, Kosus $\mathrm{N}$, et al studied transcervical intrauterine levobupivacaine and lidocaine infusion for pain control during endometrial biopsy in 99 patients. $^{4}$ The pain score in the study groups was significantly lower than the control group $(\mathrm{p}<0.001)$ however, there was no difference between the levobupivacaine and lidocaine group $(\mathrm{p}=0.641)$. Cengiz $\mathrm{H}$ et al, conducted a study on patients undergoing endometrial biopsy and randomized 67 women to the paracervical block and 53 to the intrauterine lidocaine. ${ }^{5}$ The main outcome measure was pain intensity, measured using the VAS, during and after the procedure. The pain scores immediately after the procedure were similar in the groups $(p=0.079)$ but the pain scores 30 min after the procedure were significantly lower in the intrauterine group than in the paracervical group $(\mathrm{p}=0.0001)$. In 2015, Paulander TS et al, did a study on 218 women with a topical formulation of lidocaine on cervix before intrauterine device insertion. ${ }^{6}$ Mean VAS score for maximum pain during the first 10 minutes after IUD insertion was $36 \%$ lower with lidocaine than with placebo and was also significantly lower at 30 minutes. In 2015, Aksoy $\mathrm{H}$ et al, performed a double blind RCT on 200 women and studied the role of application of $10 \%$ lidocaine spray to the cervix during IUD insertion. ${ }^{7}$ The mean pain score during the procedure was significantly lower in the lidocaine spray group as compared to the placebo group ( $p<0.001$ ). In 2015, Tavakolian $S$ et al, performed a RCT on 46 women and examined the effectiveness of local application of lidocaine/prilocaine $5 \%$ cream to the cervix in reducing pain during IUD insertion or removal. ${ }^{8}$ Pain severity was assessed using a validated $10-\mathrm{cm}$ VAS. Pain severity during the tenaculum application, insertion of uterine sound and insertion or removal of IUD was significantly lower with lidocaine/prilocaine cream compared with placebo ( $p$ $<0.001)$.

Karasu Y et al, conducted a RCT on 200 multiparous women of reproductive age and studied pain score using lidocaine spray and injection groups versus control during IUD insertion. ${ }^{9}$ They, concluded that lidocaine spray reduced pain related to both tenaculum use and IUD insertion. Spray application was easy and less time consuming. Paracervical lidocaine injection also reduced pain during IUD insertion, but had no effect on tenaculum-related pain and also the injection was painful Benchahong $\mathrm{S}$ et al, conducted a study on 250 patients undergoing EB, the subjects were randomly allocated to either receive intrauterine lidocaine (study group) or normal saline (control group). ${ }^{10}$ This study showed that there was significant pain reduction in patients who received intrauterine instillation of lidocaine compared to placebo, during the procedure as well as 15 minutes and 2 hours after procedure ( $\mathrm{p}<0.0001)$. Patient satisfaction was not significantly different between the two groups, while physician satisfaction significantly better in the lidocaine group.

In 2018, Luangtangvarodom $\mathrm{W}$ et al, conducted a study on 140 participants using topical spray of $10 \%$ lidocaine before endometrial aspiration. Topical lidocaine spray significantly relieved pain during the procedure. Both groups had no significant differences of postoperative pain at 15 minutes and 2 hours.

Data analysis proved a statistically significant decrease in pain during endometrial biopsy/IUD insertion with intrauterine levobupivacaine in women. Even though instillation is likely to extend the procedure time, the relief from pain for the patient is worthy and overcome the time factor.

\section{CONCLUSION}

Study sought to reduce pain during OPD procedures which might lead to better patient experience, reduce anxiety and produce an overall better outcome. We conclude that intrauterine instillation of $5 \mathrm{ml}$ of $0.5 \%$ of levobupivacaine, 15 minutes before the procedure significantly reduced pain during the procedure. It also reduced the need of additional analgesia. This feasible intervention during the OPD procedure help women to return to normal activity early. Intrauterine instillation of levobupivacaine had an advantage as it is easy to administer, cost-effective and comfortable to patient. So, 
intrauterine levobupivacaine is a suitable analgesic option during routine OPD gynecological procedures.

\section{Funding: No funding sources}

Conflict of interest: None declared

Ethical approval: The study was approved by the Institutional Ethics Committee

\section{REFERENCES}

1. Fothergill DJ, Brown VA, Hill AS. Histological sampling of the endometrium - a comparison between formal curettage and the Pipelle sampler. $\mathrm{Br}$ J Obstet Gynaecol. 1992;99:779-80.

2. Burlacu CL, Buggy DJ. Update on local anesthetics: focus on levobupivacaine. Ther Clin Risk Manag. 2008;4(2):381-92.

3. Akgun N, Keskin E, Kalem MN, Bakirarar B. Intrauterine levobupivacaine for pain control during intrauterine device insertion. Int J Reprod Contracept Obstet Gynecol. 2017;6(3):1117-21.

4. Kosus N, Kosus A, Demircioglu RI. Transcervical intrauterine levobupivacaine or lidocaine infusion for pain control during endometrial biopsy. Pain Res Manag. 2014;19(2):82-6

5. Cengiz H, Dagdeviren H, Kaya C, Yesil A, Caypinar SS. Comparing the efficacy of intrauterine lidocaine and paracervical block in decreasing the pain associated with endometrial biopsy: a randomised trial. Arch Gynecol Obstet. 2014;289:609-14.

6. Tornblom-Paulander S, Tingaker BK, Werner A, Liliecreutz C, Conner P, Wessel H. Novel topical formulation of lidocaine provides significant pain relief for intrauterine device insertion: pharmacokinetic evaluation and randomized placebocontrolled trial. Fertil Steril. 2015;103(2):422-7.

7. Aksoy H, Aksoy U, Ozyurt S, Acmaz G, Babayigit M. Lidocaine $10 \%$ spray to the cervix reduces pain during intrauterine device insertion: a double-blind randomised controlled trial. J Fam Planning Reprod Health Care. 2016;42(2):83-7.

8. Tavakolian S, Doulabi MA, Baghban AA, Mortazavi A, Ghorbani M. Lidocaine-prilocaine cream as analgesia for IUD insertion: a prospective, randomized, controlled, triple-blinded study. Glob J Health Sci. 2015;7(4):399-404.

9. Karasu Y, Cömert DK, Karadağ B, Ergün Y. Lidocaine for pain control during intrauterine device insertion. J Obstet Gynaecol Res. 2017;43(6):1061-6.

10. Benchahong S, Chanthasenanont A, Pongrojpaw D, Pattaraarchachai J, Bhamarapravatana K, Suwannarurk K. Efficacy of intrauterine lidocaine instillation in reducing pain during endometrial biopsy by novak. Pain Res Treat. 2018;2018:9368298.

11. Luangtangvarodom $\mathrm{W}$, Pongrojpaw D, Chanthasenanont A, Pattaraarchachai J, Bhamarapravatana K, Suwannarurk K. The efficacy of lidocaine spray in pain relief during outpatientbased endometrial sampling: a randomized placebocontrolled trial. Pain Res Treatment. 2018;2018:1-5.

Cite this article as: Gupta T, Mansinghka A, Thakur A. Role of intrauterine instillation of levobupivacaine as a local anaesthetic for outpatient gynaecological procedures: a randomized controlled study. Int $\mathbf{J}$ Reprod Contracept Obstet Gynecol 2020;9:937-41. 